\title{
Recuperación y salvaguarda del patrimonio industrial. La ex-fábrica de gas San Borja, Santiago de Chile transformada en el Parque Urbano de los Gasómetros
}

\author{
Stefania Pareti Petruccelli, Irene Kraft Gutiérrez, Blanca García-Henche
}

\begin{abstract}
Resumen: El objetivo del presente trabajo es explorar la conservación del patrimonio industrial de una ciudad a través de la regeneración urbana y el desarrollo de polos urbanos sostenibles. Se analiza el caso del Parque de los Gasómetros en Santiago de Chile, como ejemplo de revitalización del patrimonio industrial de una antigua fábrica de gas que se ha transformado en el primer barrio sustentable la ciudad.

Se efectúa una revisión de fuentes secundarias para analizar la puesta en valor que ha realizado Parque de los Gasómetros y se lleva a cabo un análisis del uso y posicionamiento de la imagen de marca del proyecto.

Se concluye que la salvaguarda del patrimonio industrial, junto con el desarrollo de la nueva marca Parque de los Gasómetros, ha sido un aporte tanto para los habitantes como para la revitalización patrimonial del barrio, desarrollando, además, la integración social y densidad equilibrada del uso de suelo.
\end{abstract}

Palabras clave: conservación urbana, antigua fábrica de gas, patrimonio industrial, marketing cultural y urbano, revitalización urbana, imagen de marca, barrio urbano sustentable, Santiago de Chile

\section{Recovery and safeguarding of industrial heritage. The ex-gas factory San Borja, Santiago de Chile, transformed into the Urban Park of Gasometers}

Abstract: The main objective of this work is to explore how industrial heritage of a city neighborhood can be preserved through urban regeneration and development of sustainable urban centers. The case of the Parque de los Gasómetros in Santiago de Chile is analyzed, as an example of revitalization of the industrial heritage of an old gas factory that has become the first sustainable neighborhood in the city.

A review of secondary sources is carried out to analyze the enhancement made by Parque de los Gasómetros and an analysis of the use and positioning of the projects brand image is carried out.

It is concluded that the safeguarding of the industrial heritage together with the development of the new Parque de los Gasómetros Brand has been a contribution to both the inhabitants and the heritage revitalization of the neighborhood, also developing the social integration and balanced density of land use.

Keyword: urban conservation, old gas factory, industrial heritage, cultural and urban marketing, urban revitalization, brand image, sustainable urban neighborhood, Santiago de Chile

\section{Recuperação e salvaguarda do património industrial. A ex-fábrica de gás San Borja, em Santiago do Chile, transformada no Parque Urbano de los Gasómetros}

Resumo: O objetivo do presente trabalho é explorar a conservação do património industrial de uma cidade através da regeneração urbana e o do crescimento dos polos urbanos sustentáveis. Analisa-se o caso do Parque de los Gasómetros em Santiago de Chile, como exemplo da revitalização do património industrial de uma antiga fábrica de gás que se transformou no primeiro bairro sustentável da cidade. 
É feita uma revisão de fontes secundárias para analisar a valorização no Parque de los Gasómetros e o uso e o posicionamento da imagem de marca do projeto

Conclui-se que a salvaguarda do património industrial, a par do desenvolvimento da nova marca Parque de los Gasómetros, tem sido um contributo tanto para os habitantes como para a revitalização do património do bairro, desenvolvendo também a integração social e a densidade equilibrada do uso da terra.

Palavras-chave: conservação urbana, antiga fábrica de gás, património industrial, marketing cultural e urbano, reabilitação urbana, imagem de marca, bairro urbano sustentável, Santiago de Chile

\section{Introducción y descripción del proyecto de investigación}

La presente investigación busca explorar la recuperación de la arquitectura industrial, analizando el caso de la Fábrica de Gas San Borja, ubicada en el Barrio de Estación Central en Santiago de Chile. Dicha fábrica se encuentra actualmente en plena recuperación y puesta en valor de su patrimonio industrial tras el proyecto Parque de los Gasómetros, lo que justifica el interés de esta investigación.

En este apartado se van a detallar las ideas que justifican el presente estudio y los objetivos de este. Seguidamente, se llevará a cabo un análisis de las tendencias de puesta en valor del patrimonio industrial para llegar a explicar el caso que nos ocupa. Posteriormente, se presenta el posicionamiento e imagen de marca como elemento de identificación urbana de barrios o geolocalizaciones, especialmente cuando se trabaja bajo el prisma de barrios sustentables como estrategia de posicionamiento.

\section{Justificación y objetivos de la investigación}

En estas últimas décadas se han realizado profundos cambios en torno al concepto de patrimonio (Aguilar 2002). Hoy día, el valor cultural e histórico de muchos edificios industriales parece que está fuera de toda duda y su conservación como testimonio de un pasado industrial, de un momento histórico determinado, dentro de un entorno determinado, es un tema del que se es consciente.

En los diferentes estudios, que se detallan posteriormente, se observa que en las investigaciones sobre patrimonio industrial resulta recurrente el tener que repensar nuevos usos para preservar el patrimonio y actualizarse a la vez a nuevos retos. Así, el desarrollo de estos proyectos requiere de una renovación arquitectónica de los edificios abandonados, la inserción de nuevos espacios atractivos que modifiquen la imagen urbana tenida hasta entonces y la implantación de usos comerciales, de habitabilidad, turísticos y de ocio que atraiga a la población foránea (Arnet 2014: 19-27), teniendo en cuenta siempre la sostenibilidad de los proyectos realizados.

En el caso de Chile existen numerosos ejemplos de regeneración urbana a través de la recuperación del patrimonio industrial, como la recuperación del Barrio
Matadero Franklin, la implantación del Centro Nacional de Arte Contemporáneo en las instalaciones del antiguo aeropuerto de Cerrillos de Santiago, el proyecto Isla Teja en Valdivia, donde se ubicaba una antigua fábrica de ladrillos y tejas de la época colonial, que es actualmente el Campus Isla Teja de la Universidad Austral de Chile, o la creación del Centro Cultural Estación Mapocho en la antigua estación ferroviaria de principios del siglo XX.

El objetivo principal del presente trabajo es explorar cómo se puede conservar el patrimonio industrial de una ciudad barrio a través de la regeneración urbana y el desarrollo de polos urbanos sostenibles. Se busca conocer cómo la salvaguarda y la puesta en valor del patrimonio industrial permiten que se mantenga un legado histórico y cultural de un barrio, facilitando también una mejor calidad de vida de la comunidad y permiten desarrollar un control de la densidad del uso de suelo en el sector.

La justificación del trabajo y selección de Parque de los Gasómetros se debe a: (1) si bien existen numerosos casos de puesta en valor y salvaguarda del patrimonio industrial de gasómetros, este es el primer caso dónde se transforma el espacio utilizado por gasómetros para crear un barrio sustentable en Chile, (2) es un ejemplo concreto de cómo una compañía privada, como Gasco en este caso, protege y salvaguarda el patrimonio industrial, (3) se observan nuevos usos de espacios, de gasómetro a barrio sustentable, donde habrá comercio local, cultura, deportes, (4) la comunidad de vecinos ha sido integrada, teniendo participación y opinión en el desarrollo del proyecto.

La hipótesis por contrastar se basa en que se puede conservar el patrimonio industrial de una ciudad barrio, a través de la regeneración urbana y el desarrollo de polos urbanos sostenibles.

\section{Puesta en valor del patrimonio industrial: Los gasómetros como recursos de patrimonio industrial}

El patrimonio tuvo su primera caracterización gracias a las construcciones $u$ objetos que eran estéticamente bellos (Rahola 1998: 12). Rahola en 1998 define el concepto de patrimonio como el de un bien histórico que sirve como objeto de estudio para conocer lo que pasó en sociedades del pasado, a través de una obtención de datos cualitativos. 
Cada país tiene su propia historia de desarrollo, en donde cada uno ha explotado distintos recursos naturales para poder crecer económicamente (Edwards y Coit 1996: 350). Este desarrollo económico ha ido parejo con el desarrollo de una arquitectura industrial para la explotación de recursos que años más tarde pasa a estar en desuso y que en numerosos casos ha sido rehabilitada, aunque en otros este patrimonio ha sido abandonado.

El patrimonio industrial se define como los restos de la cultura industrial que tiene un valor histórico, social, arquitectónico y científico, convirtiéndose en un símbolo cultural de la respectiva ciudad en la que se encuentra. El valor histórico demuestra el desarrollo y avance industrial que han alcanzado las sociedades. Además, el patrimonio industrial tiene una característica que le diferencia del patrimonio monumental y es que no es necesariamente un objeto único y singular, ya que lo que se hizo en el período de la industrialización fue homogeneizar la forma de trabajo, en donde las herramientas y técnicas se vieron estandarizadas, cuyo efecto fue un cambio en la sociedad. Gracias a esta homogenización se pudo llegar a la producción en serie y el potencial intercambio de piezas, donde los productos de una misma fábrica llegan a ser idénticos.

En la mayoría de los ejemplos de proyectos de recuperación del patrimonio industrial, el turismo supone una fuerza muy importante para la manutención de dicho patrimonio, ya que existe un interés por parte de la industria turística por generar nuevos productos y servicios (Jonsen-Verbeke 1999: 74 y Kerstetter, Confer y Bricker 1998: 93).

Dentro del patrimonio industrial de algunas ciudades se encuentran unas construcciones industriales que son grandes depósitos de almacén de gas, llamados gasómetros. Los gasómetros son estructuras cilíndricas de acero que antiguamente se utilizaban para contener el gas que permitía la iluminación nocturna de las ciudades, tanto el alumbrado como para el consumo doméstico. Cabe destacar que han sido mal llamados gasómetros, ya que no son medidores, sino sólo depósitos de gas (Tartás y Guridi 2016: 37). En el siglo XX eran símbolo de progreso y desarrollo, dado que estos gasómetros otorgaban esta nueva fuente de energía, lo que generaba un prestigio social y gran brecha entre la ciudad y el campo (Tartás y Guridi 2016: 38). Actualmente, en gran parte, han perdido su función por la aparición de nuevas y mejores técnicas y tecnologías. Hay casos en que estos gasómetros han quedado abandonados y fuera de contexto con su entorno y, por otra parte, han adquirido un significado histórico y cultural (Fiorino, Landorfo y Mazzolani 2015: 264).

Existe evidencia de cómo la salvaguarda y la revalorización del patrimonio industrial de los gasómetros puede afectar positivamente al territorio y sus comunidades. Por ello, a continuación, se muestran diferentes ejemplos de recuperación del patrimonio industrial relacionado con los gasómetros tanto en zonas o regiones concretas como en diferentes ciudades:
- Los Gasómetros de Zollverein. En Alemania, en la cuenca del Ruhr existían innumerables gasómetros, que se utilizaban como depósitos de la producción de las minas de carbón. Los que son de mayor volumen y característicos están protegidos y catalogados como monumento en la misma cuenca del Ruhr. Esta preservación y cuidado permite obtener a la región una identidad propia (Tartás y Guridi 2016: 43).

- El Gasómetro de Oberhausen de 1929 fue reconvertido en 1993 en una especie de hall de 3000 metros cuadrados para poder realizar exposiciones y en la parte superior se ubican salas de espectáculos y exposiciones (Tartás y Guridi 2016: 44).

- El Gasómetro de Lebón. En la ciudad de Valencia, España, la fábrica de Gas Lebón contiene los restos de un gasómetro que, desde 1867 durante décadas, le entregó electricidad a la ciudad. Los alrededores del gasómetro fueron destruidos para poder construir viviendas y en 2017 el Ayuntamiento de Valencia aprobó los trámites para poder restaurar y darle valor a esta antigua construcción ubicándola en un jardín de la ciudad e incluyéndola en el Catálogo Estructural de Espacios y Bienes Protegidos del Ayuntamiento como Bien de Relevancia Local (Domínguez 2020; Valencia Bonita 2020).

Como se explicaba al inicio, la presente investigación busca analizar la puesta en valor un nuevo uso que se está llevando a cabo para una ex fábrica de gas San Borja en Santiago de Chile. El barrio en donde se ubica era, en el siglo XIX, un sector agrícola cercano a Santiago. Posteriormente, se construyó, en 1857, una estación de ferrocarriles, convirtiéndose en un barrio ferroviario y urbano. Años más tarde, en 1897, se construyó la estructura metálica que cubre el sector de los andenes [figura 1] que comenzó a definir la estructura arquitectónica del barrio (véase el plano del barrio recogido en la figura 2).

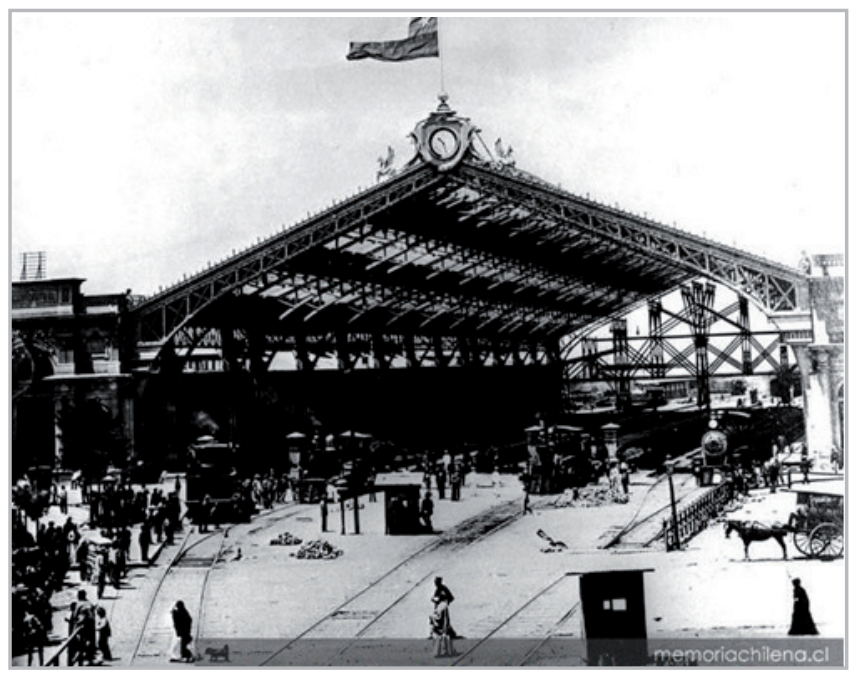

Figura 1.- Estación ferroviaria de Estación Central, construida en el año 1897. Fuente: Biblioteca Nacional de Chile, 2021. 


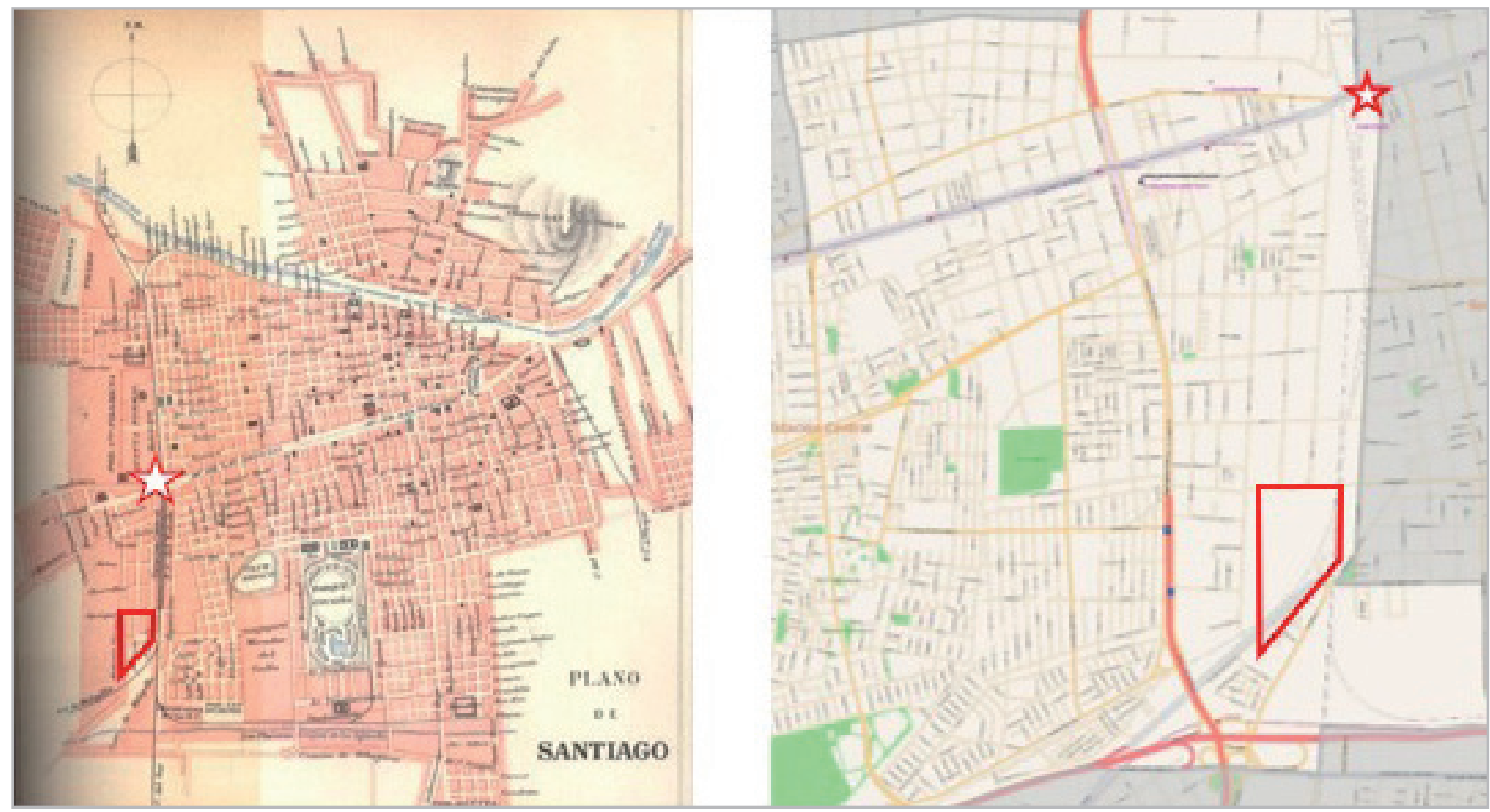

Figura 2.- Izquierda: Plano de Santiago, año 1885. Derecha: Plano comuna Estación Central año 2021. Estrella indicando Estación ferroviaria y polígono ubicación de fábrica de gas. Fuente: Biblioteca Nacional de Chile, 2021

La compañía encargada de realizar el suministro y gestión de este servicio era la compañía de alumbrado "Urmeneta e Eastman" que, en 1865, se transformó en la sociedad anónima "Compañía de Gas de Santiago". La inauguración del servicio de alumbrado fue el año 1867, iluminando lo que hoy en día sigue siendo el Teatro Municipal de Santiago. En el año 1910 nació la Fábrica San Borja [figura 3], la cual aportó, junto con la Fábrica de San Miguel, gas a la ciudad, dado que la demanda del recurso aumentó notoriamente. Luego nació el primer gasómetro de 23.000 metros cúbicos que aportaba abastecimiento a la ciudad de Santiago. Desde 1922 ya se llevaban a cabo actividades deportivas y recreativas en la fábrica, junto con establecimiento de consultorios médicos. En el año 1956 se iniciaron las plantas de inyección de gas licuado, mecanizando y automatizando las plantas de carbón y gas. En el año 2015, la Fábrica de San Borja, dio término a las operaciones industriales (Atisba 2020; Parque de los Gasómetros 2020).

No hubo ningún tipo de proyecto hasta el arribo de Empresas Gasco (empresa privada chilena del sector energético, con 160 años de historia, que comercializa el gas licuado y natural en Chile), con la idea del proyecto Parque de los Gasómetros 2020, cuya finalidad es aportar valor al legado de la fábrica de manera integral y sostenible.
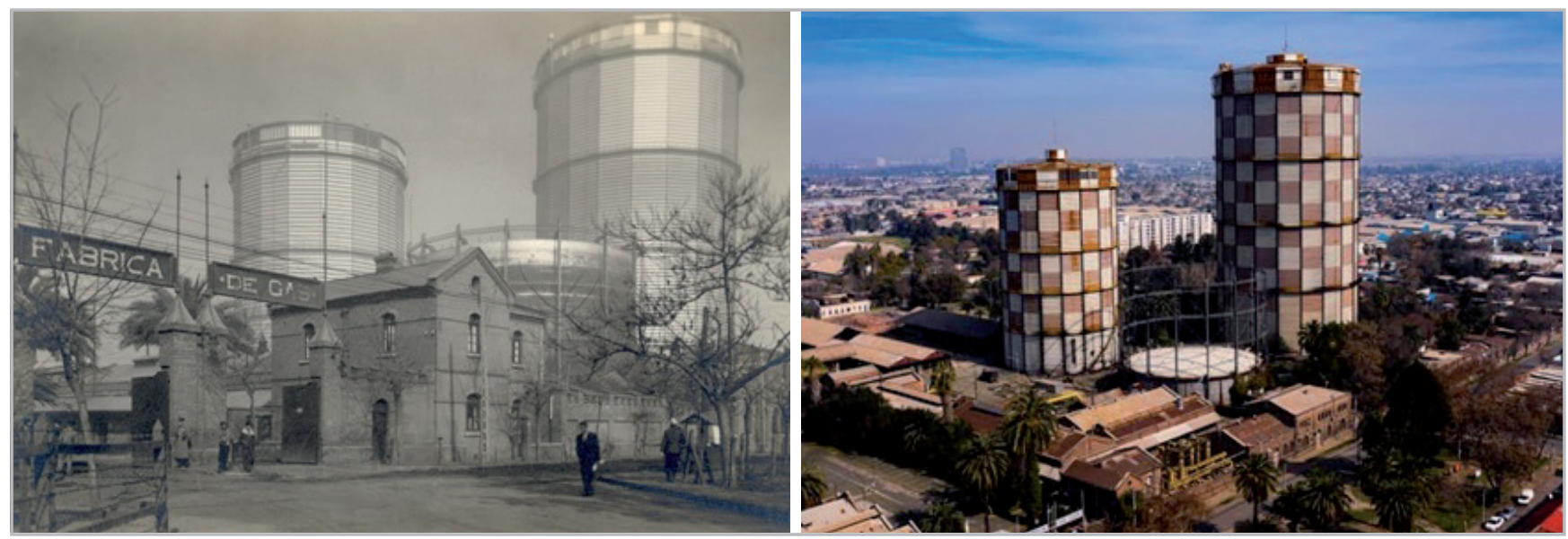

Figura 3.- Fábrica de gas San Borja (año 1910 y actualidad). Fuente: Sitio oficial Parque de los Gasómetros en la red social Facebook, 2020. 
En el año 2019 se presentó el proyecto Parque de los Gasómetros, dirigido por Empresas Gasco y la oficina de urbanismo Atisba, cuyo objetivo es poner en valor y salvaguardar a la antigua Fábrica de San Borja. El proyecto busca transformar el terreno abandonado en un lugar rodeado de áreas verdes para poder recorrer y disfrutar, junto con reconvertir algunas de las instalaciones en nuevos espacios para comercio local (CAR), puestos de comida o artesanías y distintos servicios para la comunidad (servicios para deporte y cultura, oficinas, etc., tal como recoge la figura 4).

Por otra parte, el proyecto Parque de Gasómetros tiene como fin que la sociedad pueda conectarse de alguna manera con el pasado, con lo que sucedió en la ciudad, dándole vida a los gasómetros y destacarlos como iconos urbanos (Atisba 2020; Parque de los Gasómetros 2020).

En el sitio oficial de Facebook del proyecto se pueden rescatar las siguientes citas extraídas de parte de su equipo directivo y de comunicaciones:
- "En Parque de los Gasómetros queremos desarrollar un nuevo barrio con espacios para vivir en torno a áreas verdes y sus gasómetros".

-“En el pasado, la ex Fábrica era un lugar de encuentro para la comunidad. En Parque de los Gasómetros queremos recuperar esos espacios para los vecinos, a través de un área destinada a actividades recreativas y deportivas".

- “Queremos que los vecinos de Estación Central tengan comercio y servicios cerca de los lugares donde viven. Por eso, Parque de los Gasómetros considera una zona con tiendas y almacenes con escala de barrio, además de espacios para trabajar".

Dichas citas ponen el foco en la revitalización del área desde el punto de vista del diseño de una oferta habitacional, además de lúdica y cultural, intentando la generación de una nueva identidad del área bajo la marca Parque de Gasómetros, lo cual se consigue a través de la constante información en redes y medios de comunicación de los diferentes aspectos del proyecto.

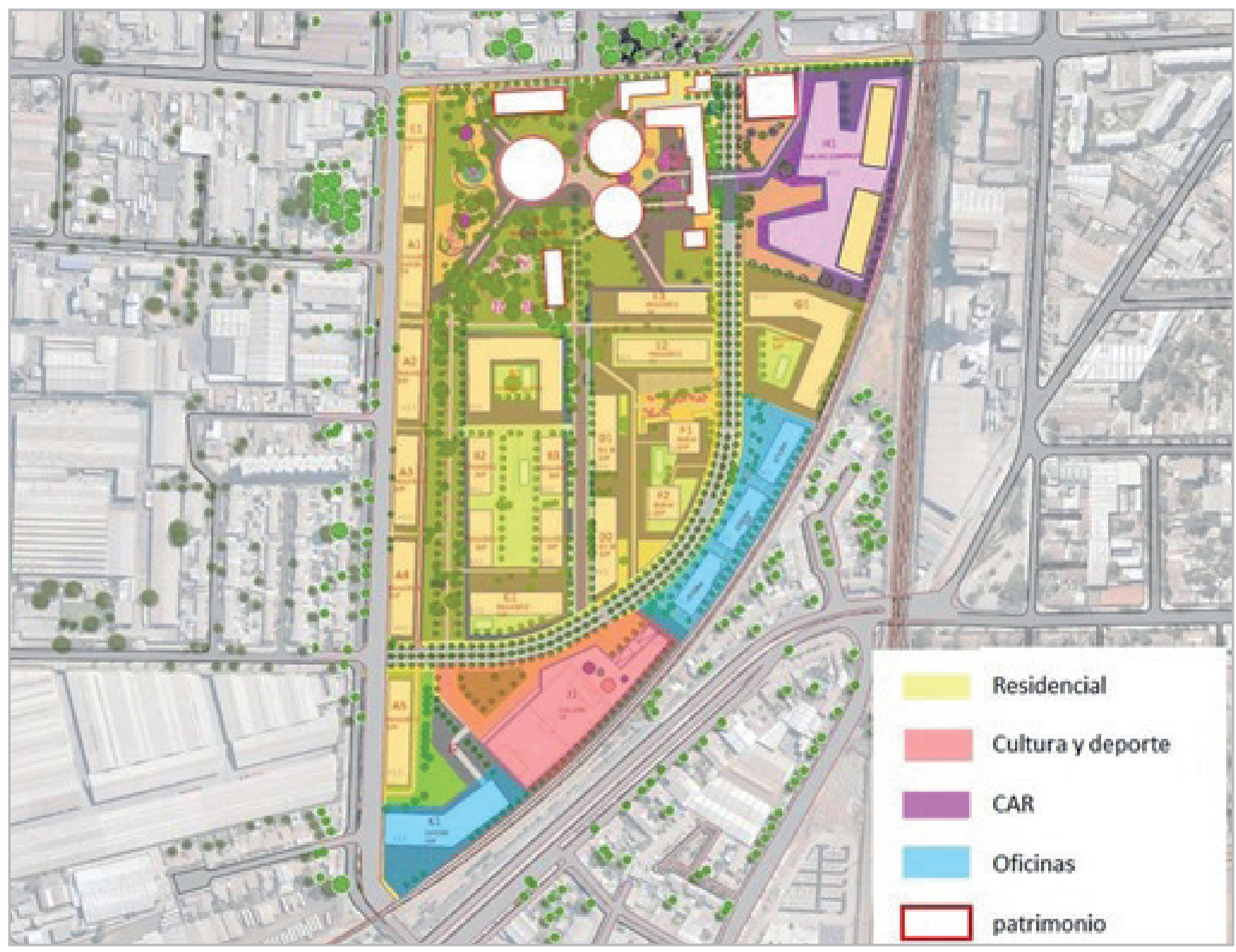

Figura 4.- Mapa de proyecto Parque de los Gasómetros en Estación Central. Fuente: Página oficial Parque de los Gasómetros en Facebook, 2020. 


\section{Posicionamiento e imagen de marca como elemento de identificación urbana de barrios o distritos o geolocalizaciones}

El concepto Imagen de Marca representa un importante aspecto en las actividades de marketing, siendo un activo que refuerza el valor de los productos, utilizado por las organizaciones para poder diferenciarse de sus competidores (Martínez et al 2005: 91; Ogba y Tan 2009: 133). Estas marcas creadas por las organizaciones o instituciones van evolucionando junto a ellas, pasando por diferentes cambios, pero llegan al punto de ser un valor de la misma empresa (Orozco y Roca 2011: 275).

Diferentes autores confirman que la creación de una imagen de marca, junto con una actitud de marca, ayuda a mejorar el valor de la marca misma, además de una gran satisfacción proveniente del consumidor y una posible lealtad del cliente/visitante (Faircloth et al 2001: 70; Ogba y Roca: 134). Se ha de considerar, por otra parte, que, la imagen de marca no es una construcción interna de la empresa, sino que es una materialización de lo que es percibido por una construcción mental de, por ejemplo, grupos de interés, por lo que es algo subjetivo (Orozco y Roca 2011: 275). Representan las percepciones emocionales o racionales de los consumidores, donde la calidad que se percibe es lo que el cliente está percibiendo de la calidad general de un producto/servicio (Ogba y Tan 2009: 134).

Los territorios con valor patrimonial han de generar diferenciación y, para ello, es importante trabajar en el posicionamiento e identificación de estos por parte del público objetivo al que se dirigen (Coca-Stefania 2014). A través del estudio realizado en Parque de Gasómetros se va a analizar la importancia de la generación de identidad del proyecto proponiendo beneficios sociales y psicológicos en la identidad de quienes componen el proyecto $y$, a su vez en la percepción de quienes lo visitan o disfrutan, dándole un sello único e imposible de replicar debida a la diferenciación e imagen de marca desarrollada.

En el marketing urbano una marca destino es el nombre del lugar y dichos destinos han de trabajar con el fin de crear una imagen de marca positiva e identificativa del lugar. En el caso del Parque de Gasómetros el nombre identifica, claramente, el origen industrial de la zona donde se desarrolló una actividad industrial en la ciudad creando así una identidad e imagen basada en el origen histórico, industrial y obrero de la zona.

\section{Barrio Sustentable como componente de la identidad de marca de un lugar}

Muchas ciudades se han centrado en la última década en buscar modelos estratégicos que les permitan un desarrollo económico y social sostenible. Son ejemplos destables el proyecto de recuperación y planificación urbana del barrio Wesltern Harbour, un parque industrial degradado del puerto de en Malmö en el sur de Suecia o el proyecto 22@ barcelona que reinterpreta la función de antiguos tejidos industriales del barrio de Poblenou. Teniendo en cuenta la planificación integral y las políticas urbanas, estos ejemplos de proyectos, entre otros muchos, suponen una experiencia para tener en cuenta por ciudades de América Latina, que están sufriendo numerosos cambios urbanos y sociales en la actualidad.

La justificación de la presente investigación se fundamenta en que no existen, hasta la fecha, numerosas metodologías sistemáticas para gestionar adecuadamente patrimonio cultural industrial junto a desarrollo territorial y urbano sustentable (Bandarin y Van Oers 2012).

Las sociedades han decidido empezar por un diseño responsable y ecológico de los barrios, lo cual lleva al concepto de urbanismo y arquitectura sustentables. Estos conceptos incluyen el manejo de recursos naturales, económicos y humanos de manera responsable y ambientalmente amigable con el entorno (Hernández 2008: 301-302). Todo esto se debe al crecimiento exponencial de las poblaciones y ciudades, que ha llevado a una sobreexplotación de recursos naturales por parte de la población (Shi et al 2016: 973) y, además, a su pérdida de identidad.

En el último tiempo se ha estado revirtiendo el daño implementando una serie de programas de recuperación de barrios en Chile, siendo uno de los programas más exitosos "Quiero mi barrio", del Ministerio de Vivienda y Urbanismo (MINVU), el cual tiene un foco orientado la mejora de los barrios de manera integral, devolviéndole así la identidad a las poblaciones y respondiendo ante las necesidades de la comunidad y del medio ambiente (Blanco 2015: 210; Link et al 2017: 12; Valenzuela et al 2009: 286). La misma creación de barrios sustentables puede mejorar los diferentes ambientes que se dan en las localidades dado su actual contaminación y deterioro por el crecimiento urbano (Shi et al 2016: 973).

La iniciativa "Ciudades Saludables" lanzada por la Organización Mundial de la Salud (OMS) pretende crear asentamientos más sustentables y ambientalmente amigables. Aparece así el concepto de Barrio sustentable o Barrio sostenible, concepto que proviene de la traducción del inglés de "sustainable neighborhood", considerando que las ciudades que trabajan en la construcción de dichos barrios conseguirán diversas ventajas tales como la reconstrucción y rescate de terrenos o sitios degradados o abandonados (Blanco 2015: 74-75), la mejora en la gestión de las ciudades y promoción del cuidado hacia el medio ambiente y la calidad de vida. Esa diferenciación consigue posicionar a ciudad y barrios bajo una imagen relacionada con la sostenibilidad de la que muchos lugares del mundo se benefician por su posicionamiento y diferenciación relacionada con la ubicación, la energía utilizada, la puesta en valor de zonas industriales degradadas etc., como ocurre en el barrio La Pinada en Valencia (único 
proyecto español seleccionado para formar parte de Smart Sustainable Districts) o Mammarby en Stocolmo (Proyecto de reconversión de una zona industrial en el primer barrio eco-friendly Estocolmo).

Primož Medved (2017), define los ejes comunes en los que han de basarse el desarrollo de proyectos de barrios sostenibles:

- Transitabilidad a pie.

- Conexiones: las calles bien conectadas dispersan el tráfico y facilitan el paso a peatones.

- Variedad de edificaciones y usos: diferentes tipos de viviendas, oficinas y locales comerciales.

- Arquitectura de calidad y diseño urbanístico: énfasis en la estética y el confort humano.

- Estructura tradicional de barrio: espacio público en el centro, así como una delimitación muy clara de los límites del barrio y la zona central.

- Incremento de la densidad: más edificios, residencias, tiendas y servicios hacen del barrio un lugar interesante donde vivir.

- Transporte ecológico: movilidad sostenible basada en autobuses, tranvías y trenes.

- Sostenibilidad: buscar el mínimo impacto medioambiental en el desarrollo urbano.

- Calidad de vida: todas estas opciones facilitan un estilo de vida enriquecedor y agradable.

El término "barrio sustentable" en Chile se encuentra en desarrollo, mediante estrategias activas que quieren avanzar hacia barrios sustentables. Una de estas iniciativas es el Parque de Gasómetros, donde se plantea un cambio de uso de suelo que permita construir viviendas y áreas verdes. El plan considera transformar estas casi 18 hectáreas en un barrio habitacional que integre comercio y servicios para la comunidad. La idea es que exista una escala de barrio, con tiendas y almacenes. Además, contaría con zonas para fomentar el deporte y la cultura, conservando y potenciando los gasómetros como elementos distintivos de la historia de la comuna y la ciudad. Por otra parte, un tercio del terreno sería destinado para viviendas, un $20 \%$ de las cuales serían de integración social para familias de la comuna.

Este proyecto nace ya bajo la marca de "barrio sustentable" como estrategia para posicionar y diferenciar el proyecto y como forma de identificación de este frente a otros proyectos urbanísticos de la ciudad basándose en conceptos como sostenibilidad, integración social, áreas verdes, densidad equilibrada o revitalización patrimonial (véase figura 5, que
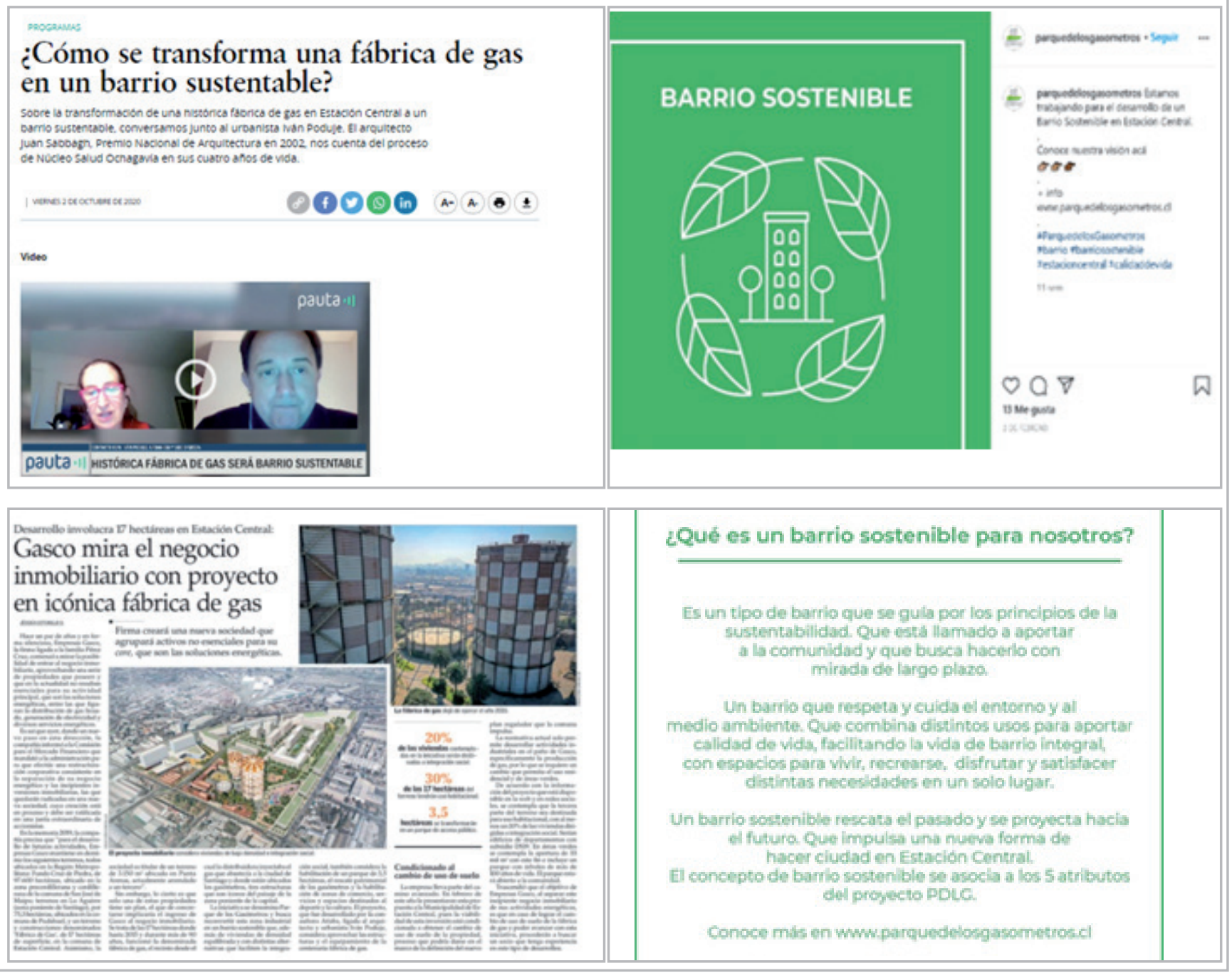


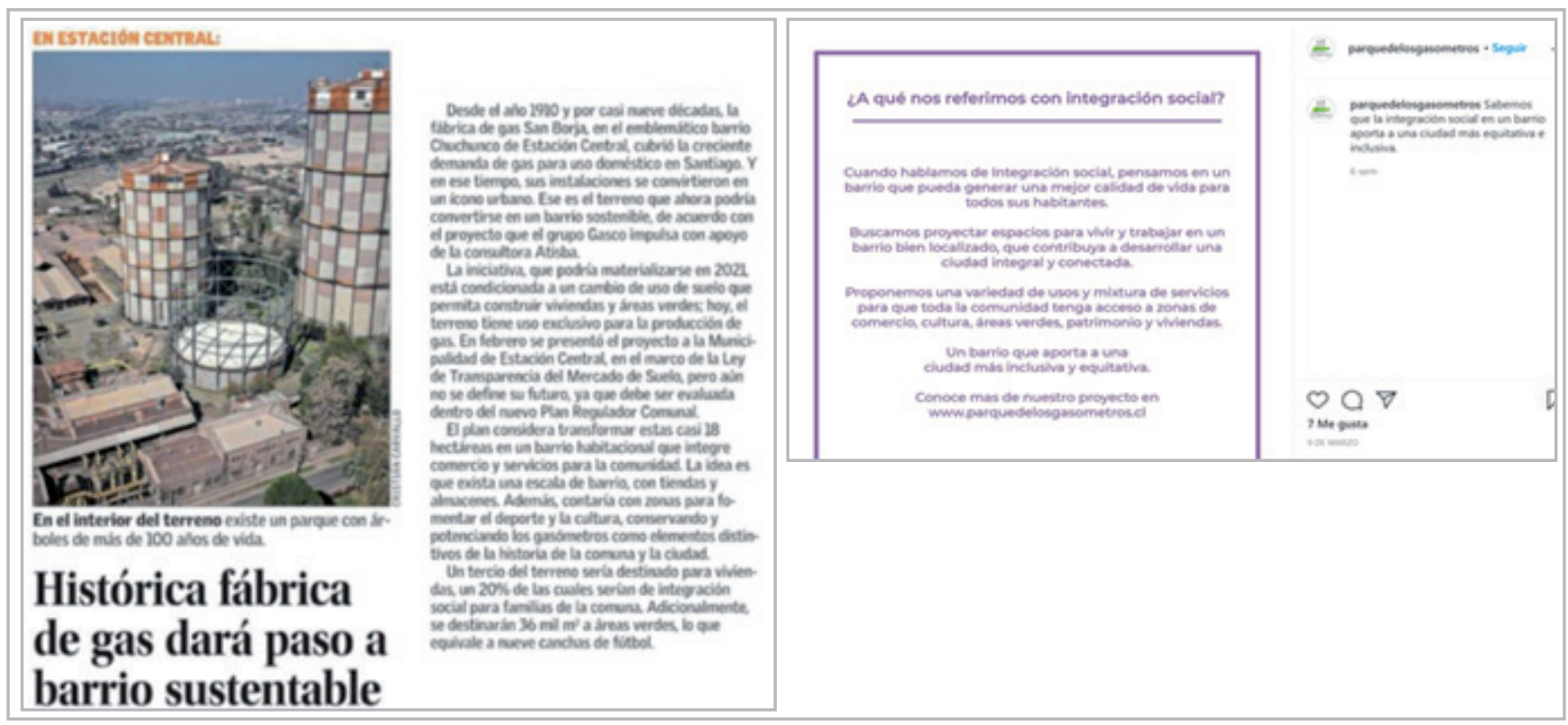

Figura 5.- . Información en medios y redes en torno al posicionamiento de Parque Los Gasómetros como barrio sostenible/sustentable. Elaboración propia a partir de página de Instagram @parquedelosgasometros y fuentes de periódicos de Pauta FM y diario El Mercurio, 2020.

recoge post de la página de Instagram del proyecto y noticias sobre el proyecto que hacen referencia a dichos conceptos).

\section{Metodología}

Como se ha indicado con anterioridad, el objetivo de la presente investigación busca comprender los efectos de la recuperación y puesta en valor del patrimonio industrial $y$ el uso de estrategias de marketing cultural y urbano relacionadas con la marca y el posicionamiento de dichos espacios como espacios sostenibles a nivel urbano. Para ello se revisa la literatura sobre recuperación del patrimonio industrial bien a través de su consolidación, recuperando su primitivo aspecto e imposibilitando su desaparición o bien a través de su renovación o reutilización para otros usos.

Se ha iniciado la exploración de la literatura con una revisión de todo el material oficial público de Parque de los Gasómetros. Por otra parte, se ha complementado la revisión anterior con un análisis notas de prensa y noticias vinculadas con el proyecto en un periodo concreto del año 2020, con el fin de crear una nube de palabras respecto a la imagen de marca que posee el proyecto. El objetivo de la creación de la nube de palabras es comprender el posicionamiento o la imagen de la marca "Parque de los Gasómetros" en la comunidad, representada a través de los medios de prensa.

La plataforma utilizada para la creación de la nube de palabras ha sido https://www.nubedepalabras.es/. Se realizó una búsqueda y recopilación de informes de medios de prensa, los que se han referido al proyecto en cuestión, para luego hacer un análisis de los titulares vinculados. En la tabla 1 se indican las fuentes. Esta técnica de creación de una nube de palabras permite tener una percepción global de las ideas principales de lo que se está analizando, para este caso concreto se han seleccionado los más representativos en base a sus fuentes editoriales [figura 6]. Se ha llevado a cabo un análisis exhaustivo del contenido, se han eliminado los conectores de cada frase y oraciones, para sólo centrar el análisis en palabras clave.

El criterio de búsqueda de titulares ha utilizado los siguientes filtros: 1) Sólo se han seleccionado titulares que hagan mención del Parque de los Gasómetros; 2) Las palabras clave de búsqueda han sido: Parque de los Gasómetros, 2021; 3) Únicamente se seleccionaron titulares dentro de las dos primeras páginas de búsqueda del motor de búsqueda arrojado por el buscador Google y 4) Exclusivamente se han considerado las noticias y titulares aparecidas en periódicos nacionales.

\begin{tabular}{|c|c|}
\hline Titular & Fuente \\
\hline $\begin{array}{c}\text { ¿Cómo se transforma una } \\
\text { fábrica de gas en un barrio } \\
\text { sustentable? }\end{array}$ & $\begin{array}{c}\text { Página principal Parque de } \\
\text { los Gasómetros. https:// } \\
\text { parquedelosgasometros.cl/parque- } \\
\text { de-los-gasometros/ }\end{array}$ \\
\hline $\begin{array}{c}\text { Gasco mira el negocio } \\
\text { inmobiliario con proyecto } \\
\text { en icónica fábrica de gas. }\end{array}$ & $\begin{array}{c}\text { El Mercurio, Economía y Negocios. } \\
\text { https://parquedelosgasometros. } \\
\text { cl/gasometros-parque-de-los- } \\
\text { gasometros-fabrica-de-gas-gasco-el- } \\
\text { mercurio/ }\end{array}$ \\
\hline $\begin{array}{c}\text { Nodo Metropolitano: } \\
\text { Gasómetros de las artes } \\
\text { circenses y teatrales. }\end{array}$ & $\begin{array}{c}\text { Proceso, Facultad de Arquitectura } \\
\text { y Urbanismo, Universidad de Chile. } \\
\text { https://proceso.uchilefau.cl/2015/ } \\
\text { project/nodo-metropolitano- } \\
\text { gasometros-de-las-artes-circences-y- } \\
\text { teatrales/ }\end{array}$ \\
\hline
\end{tabular}

Tabla 1.- Ejemplos de notas de prensa respecto al proyecto Parque de los Gasómetros. Elaboración propia. 


\section{Resultados}

Se han analizado gráficamente los conceptos claves extraídos de las notas de prensa seleccionadas estratégicamente en base a su fuente editorial [figura 6]. Al revisar la nube de palabras de las notas y noticias analizadas, se extraen los conceptos principalmente asociados al proyecto, como barrio, gas, fábrica, proyecto, terreno, cambio, comuna (ayuntamiento), viviendas, gasómetros, Estación Central, Gasco y zona. Resultan ser los tópicos más utilizados y relevantes al crear contenido de prensa sobre lo qué es el proyecto y su posicionamiento. La lógica de selección de esta técnica viene dada debido a que se permite observar gráficamente las opiniones de los diversos medios de prensa respecto a la iniciativa, por ende, se amplía la mirada más allá de lo que sólo dice el equipo organizador en sus canales de comunicación oficiales. Con todo ello se puede afirmar que se consigue un análisis neutral de la información generada para dar visibilidad al proyecto, incorporando los diversos puntos de vista y opiniones respecto al proyecto en cuestión.

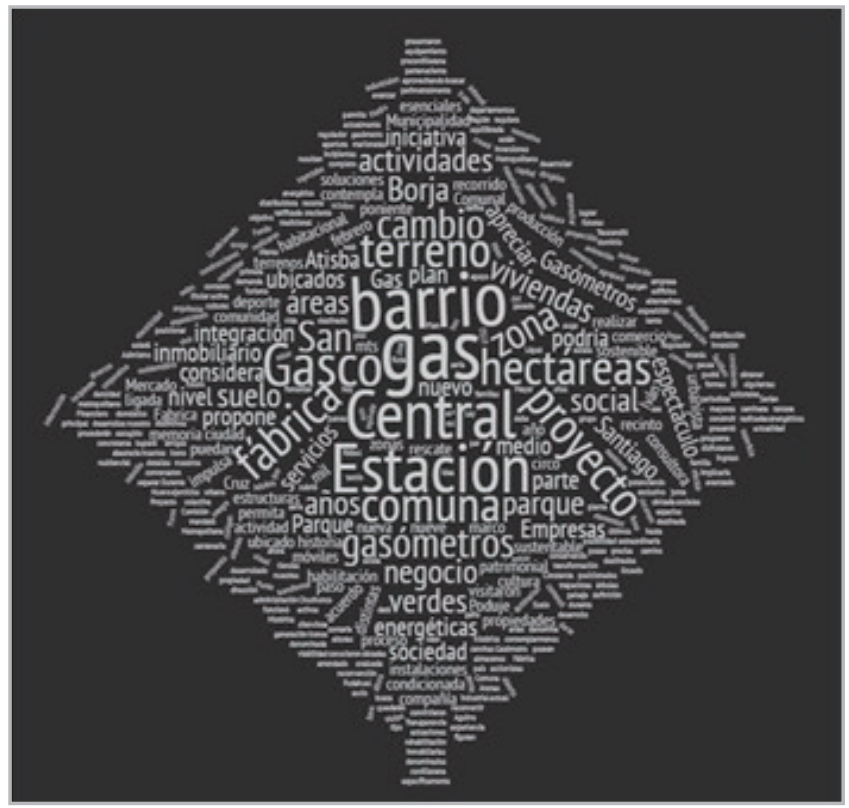

Figura 6.- Nube de palabras correspondientes a la recopilación de artículos de prensa disponibles sobre el proyecto Parque de los Gasómetros. Elaboración propia a través de nubedepalabras.es
Posteriormente se identificaron las plataformas digitales utilizadas por el proyecto con fin de potenciar la imagen de marca, a través de la comunicación y difusión. El proyecto utiliza como medio de comunicación formal las redes sociales de Facebook, cuenta creada el 17 de septiembre del año 2020, e Instagram [tabla 2], cuenta creada el 20 de agosto del año 2020. Se observa un mayor uso en la plataforma de Instagram.

Además, cuentan con sitio web oficial, el cual contiene información que se refiere a tópicos que inician desde de qué trata el proyecto, hasta la historia de los gasómetros y la opción de tomar contacto con los responsables para recibir más información (https://parquedelosgasometros. cl/).

\begin{tabular}{|c|c|c|}
\hline $\begin{array}{c}\text { Red Social } \\
\text { utilizada }\end{array}$ & $\begin{array}{c}\text { Número de } \\
\text { publicaciones a la } \\
\text { fecha (abril 2021) }\end{array}$ & $\begin{array}{c}\text { Número de seguidores } \\
\text { a la fecha (abril 2021) }\end{array}$ \\
\hline Instagram & 84 & 548 \\
\hline Facebook & 22 & 122 \\
\hline
\end{tabular}

Tabla 2.- Redes sociales utilizadas por el proyecto. Elaboración propia a partir de páginas de Facebook e Instagram (abril 2021)

El proyecto cuenta con logotipo oficial que fortalece el desarrollo de su imagen de marca y permite a la comunidad identificar en concreto al nuevo barrio Parque de los Gasómetros. Este logotipo consiste en dos gasómetros con una base verde que da a entender que es un área verde que rodea estas dos construcciones. Es un diseño simple que transmite la idea principal del proyecto, que es la revitalización de los gasómetros [figura 7].

Los resultados del análisis de las fuentes secundarias, anteriormente analizadas en las nubes de palabras, muestran las diferentes funciones que ha de cumplir una marca, en este caso Parque de los Gasómetros:
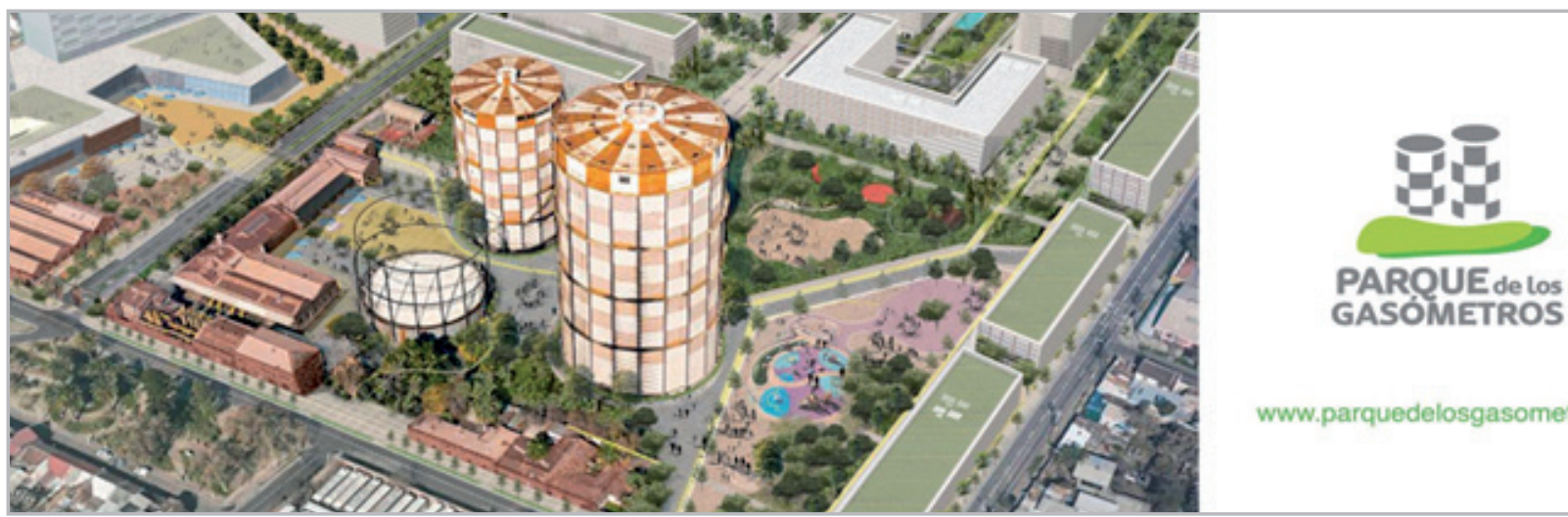

Figura 7.- Plano y logotipo oficial del proyecto Parque de los Gasómetros. Fuente: Sitio oficial https://parquedelosgasometros.cl/, 2020 
- Identificación de la zona o barrio: En el caso de Barrio San Borja Parque de los Gasómetros, la delimitación geográfica resulta clara.

- Valor de la personalización: es el primer caso a nivel latinoamericano en poner en valor el patrimonio industrial de un antiguo gasómetro, para transformarlo en barrio sustentable.

- Utilidad de selección por parte de los usuarios: Barrio San Borja, está en proceso de transformación para convertirse en un barrio sustentable.

- Garantía: La marca cuenta con el apoyo y respaldo de la compañía Gasco, la cual busca salvaguardar, escuchar opiniones de las consultas a vecinos de la comunidad.

- Valor Lúdico: Tendrá una sección especialmente dedicada a cultura y deportes.

Por otra parte, Parque de los Gasómetros como proyecto, desde sus inicios ha buscado la cercanía y estar abierto a la comunidad. Esto viene reflejado en algunas de las acciones concretas que se han llevado a cabo respecto a vinculación con los habitantes de Estación Central (Facebook Oficial Parque de los Gasómetros 2021), como realización de talleres diagnóstico que realiza el proyecto con la Junta de Vecinos $N^{\circ} 1$ Patria Nueva, Comité de Adelanto Manuel Thompson y otros entes, la invitación a la Asociación de Oficinas de Arquitectos de Chile a visitar la ex fábrica de gas o la invitación a representantes de La Ruta Mayor (programa que apoya emocionalmente a vecinos y vecinas mayores de 60 años).

\section{Conclusiones}

Uno de los principales proyectos de recuperación del patrimonio industrial es la reconversión o reutilización de dicho patrimonio para usos distintos al original, tal como ha ocurrido en el caso de estudio de esta investigación: el Parque de los Gasómetros en Santiago de Chile.

El presente trabajo estudia cómo, a través del desarrollo estratégico de un barrio sustentable en un barrio con un patrimonio industrial puesto en valor se puede fortalecer la imagen de marca de barrio, que anteriormente no poseía ningún desarrollo relacionado con sostenibilidad ni cultura desarrollo artístico. El presente artículo estudia, así, el Parque de los Gasómetros como caso de recuperación y puesta en valor del patrimonio industrial de una antigua fábrica de gas que se ha transformándose en el primer barrio sustentable de Santiago de Chile.

Se ha analizado la imagen de marca que está desarrollando el nuevo barrio, a través de análisis de contenidos extraídos de una nube de palabras que ha sido confeccionada con medios de prensa en base a parámetros previamente establecidos, junto con análisis del logotipo oficial y algunos comentarios comunicados por el Parque de los Gasómetros a la comunidad a través de sus redes sociales oficiales. Los principales resultados del contenido extraído del análisis de nube de palabras concluyen que la imagen del proyecto está asociada a las ideas de barrio, proyecto, cambio o comuna (palabras relacionadas con lo que se considera un barrio sostenible a nivel social, especialmente).

En lo que respecta a la creación de imagen, en base a los parámetros previamente definidos para el análisis de titulares, se observa que la presencia mediática ha sido alta, que Parque de los Gasómetros Barrio se ubica en una zona geográfica delimitada, posee alto valor de personalización, presenta utilidades para usuarios y visitantes, una garantía de marca sostenible y posee un logotipo oficial y una marca diferenciada.

Se concluye que la salvaguarda del patrimonio industrial junto con el desarrollo de la nueva marca Parque de los Gasómetros está siendo un aporte a tanto para los habitantes como para la revitalización y conservación patrimonial de una ex-fábrica que por años estuvo en desuso, desarrollando a su vez integración social y densidad equilibrada en el uso de suelo.

El trabajo empírico representa un caso práctico y se prueba en un caso único. Para superar esta limitación, la investigación futura podría incluir otros casos para comparar y validar nuestro modelo en otros proyectos diferentes. El análisis realizado en Parque de los Gasómetros se puede replicar en otros distritos o áreas con patrimonio industrial a recuperar, lo que representa una contribución desde el punto de vista metodológico.

\section{Referencias}

AGUILAR, I. (2002). Arquitectura industrial, testimonio de la era de la industrialización. Bienes Culturales. Revista del Instituto del Patrimonio Histórico Español, 7: 71-103.

ARNET, V. (2014). El patrimonio industrial como elemento reactivador de las ciudades intermedias chilenas. El caso de Valdivia. Revista Diseño Urbano y Paisaje, 10 (27): 19-28.

ATISBA (2020). Histórica fábrica de gas dará paso a barrio sustentable. http://www.atisba.cl/2020/09/historica-fabrica-degas-dara-paso-a-barrio-sustentable/

BANDARIN, F. Y VAN OERS, R. (2012). The Historic Urban Landscape: Managing Heritage in an Urban Century. Chichester: WileyBlackwell, 280.

BIBLIOTECA NACIONAL DE CHILE. El Barrio Estación Central (1857). Memoria Chilena. Disponible en http://www.memoriachilena.gob.cl/602/w3-article-31425.html\#imagenes. [Consulta: 16/1/2021].

BLANCO, J. P. (2015). Panorama teórico y tendencias contemporáneas de Barrios Sustentables. Especulaciones en torno a un modelo de desarrollo para Chile. Revista de Urbanismo. Santiago de Chile. 32: 70-86. 
COCA-STEFANIAK, J. A. (2014). Place branding and city centre management: Exploring international parallels in research and practice. Journal of Urban Regeneration and Renewal 7(4): 363369.

DOMínGUEZ, M. (2020). La torre del gas Lebón saldrá de su decadencia. Editorial Prensa Valenciana, S.A. https://www. levante-emv.com/valencia/2020/11/08/torre-gas-lebon-saldradecadencia-22546417.html

EDWARDS, J. A. Y COIT, J. C. L. (1996). Mines and quarries. Annals of Tourism Research, 23(2): 341-363. https://doi.org/10.1016/01607383(95)00067-4

EL MERCURIO (2020). Gasco mira el negocio inmobiliario con proyecto en icónica fábrica de gas. Parque de los Gasómetros. https://parquedelosgasometros.cl/gasometros-parque-de-losgasometros-fabrica-de-gas-gasco-el-mercurio/

\section{EMPRESAS GASCO (2019). http://www.empresasgasco.com/}

FAIRCLOTH, J. B., CAPELLA, L. M. Y ALFORD, B. L. (2001). The Effect of Brand Attitude and Brand Image on Brand Equity. Journal of Marketing Theory and Practice, 9(3): 61-75. https://doi.org/10.108 0/10696679.2001.11501897.

FIORINO, L., LANDOLFO, R. Y MAZZOLANI, F. M. (2015). The refurbishment of gasometers as a relevant witness of industrial archaeology. Engineering Structures, 84: 252-265. https://doi. org/10.1016/j.engstruct.2014.11.035

JONSEN-VERBEKE, M. (1999). Industrial heritage: A nexus for sustainable tourism development. Tourism Geographies, 1(1): 70-85. https://doi.org/10.1080/14616689908721295

KERSTETTER, D., CONFER, J. y BRICKER, K. (1998). Industrial Heritage Attractions: Types and Tourists. Journal of Travel and Tourism Marketing, 7(2): 91-104. https://doi.org/10.1300/ j073v07n02 05

LINK, F., MORA, R., GREENE, M. Y FIGUEROA, C. (2017). Patrones de sociabilidad en barrios vulnerables: dos casos en Santiago, Chile. Revista Bitácora Urbano Territorial, 27(3): 9-18. https://www. redalyc.org/articulo.oa?id=748/74853485002

MARTínEZ, E., GUTIÉRREZ, T. Y PINA, J. (2005). Propuesta de medición de la imagen de marca: un análisis aplicado a las extensiones de marca. RAE: Revista Asturiana de Economía, 33 : 89-112.

MEDVED, P. (2017). Leading sustainable neighbourhoods in Europe: Exploring the key principles and processes. Urbani Izziv 28(1): 107-121. https://doi.org/10.5379/urbani-izziven-2017-28-01-003

\section{NUBE DE PALABRAS. http://www.nubedepalabras.es}

OGBA, I. Y TAN, Z. (2009). Exploring the impact of brand image on customer loyalty and commitment in China. Journal of
Technology Management in China, 4(2): 132-144. https://doi. org/10.1108/17468770910964993

OROZCO, J. Y ROCA, D. (2011). Construcción de imagen de marca y reputación a través de campañas publicitarias de RSC. Sphera Pública, (11): 273-289. https://www.redalyc.org/articulo. oa?id=297/29729580016

PARQUE DE LOS GASÓMETROS (2020). ¿Cómo se transforma una fábrica de gas en un barrio sustentable? https:// parquedelosgasometros.cl/parque-de-los-gasometros/

RAHOLA, E. (1998). Recuperación y uso del patrimonio industrial. Ábaco, (19): 11-18. http://www.jstor.org/stable/20796398

SHI, Q., YU, T., ZUO, J. Y LAI, X. (2016). Challenges of developing sustainable neighborhoods in China.Journal of Cleaner Production, 135: 972-983. https://doi.org/10.1016/j.jclepro.2016.07.016

TARTÁS, C. Y GURIDI, R. (2016). “Los gasómetros del Zollverein: historia de una recuperación singular". Cuaderno de Notas, 0(17): 37-48. https://dx.doi.org/10.20868/cn.2016.3481

VALENCIA BONITA (2020). Valencia recuperará los restos del gasómetro del jardín del Parque Gas Lebon. https://www. valenciabonita.es/2017/09/22/el-gasometro-del-jardin-delparque-gas-lebon-de-valencia/. [Consulta: 18/12/2020].

VALENZUELA, L., JUSTINIANO, C., ARAOS, C. Y KATZ, K. (2009). Sustentabilidad en espacios colectivos de barrios vulnerables: Lineamientos para una política de espacios públicos, directrices de gestión, diseño y mantenimiento. Boletín CF+S 42/43: 285-299.

\section{Autor/es}

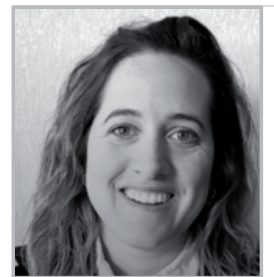

Stefania Pareti Petruccelli

s.pareti@uandresbello.edu

Facultad de Economía y Negocios. Universidad Andrés Bello, Chile

Doctora en Economía y Gestión de Empresas de la Universidad de Alcalá. Reside en Santiago de Chile y dentro de Chile se desempeña como Investigadora y docente de la Facultad de Economía y Negocios de la Universidad del Desarrollo y Andrés Bello Chile, en programas de Pre y Postgrado. Es docente también en la Universidad Diego Portales para la Facultad de Comunicaciones y Letras. Es docente además de ESAN Business School, Perú. Su línea de investigación principal es Cultura y Patrimonio, Geografía Humana, Marketing y Urbanismo. Ha sido invitada como Investigadora Visitante a la Facultad de Economía Geográfica de la Universidad de Leibniz en Hannover Alemania y a la Facultad de Arquitectura y Urbanismo de la Universidad de Bauhaus en Weimar Alemania. Pertenece al grupo de Investigación NEO Research Group de la Pontificia Universidad Católica del Perú, es Investigadora Asociada del Instituto de Investigaciones Socioeconómicas, IISEC- 
UCB. Universidad Católica Boliviana San Pablo, La Paz. Participa activamente como expositora en diversos congresos de carácter internacional centrados en temas de Conservación, Patrimonio, Cultura y Urbanismo.

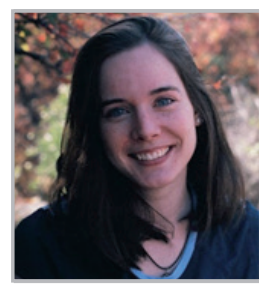

\section{Irene Kraft Gutiérrez}

i.kraftgutierrez@gmail.com

Facultad de Agronomía e Ingeniería Forestal, Pontificia Universidad Católica de Chile
Artículo enviado el 20/01/2021 Artículo aceptado el 13/05/2021

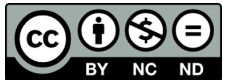

https://doi.org/10.37558/gec.v19i.889

Investigadora egresada de Agronomía e Ingeniería Forestal, con la especialidad Gestión Ambiental, en la Pontificia Universidad Católica de Chile. Habla tres idiomas: inglés avanzado, español y alemán nativo. Realizó su práctica profesional en la consultora ambiental Centro de Ecología Aplicada Ltda. Actualmente trabaja como ayudante corrector de cursos de Marketing en Facultad de Ingeniería, Escuela de Ingeniería Civil Industrial y Facultad de Economía y Negocios, Escuela de Ingeniería Comercial, Universidad Andrés Bello. Ha sido asistente en terreno de proyectos de ecología con roedores en la Reserva Nacional los Queules (Región del Maule) y guanacos en Altos de Cantillana (Región Metropolitana) (Universidad de Chiley Pontificia Universidad Católica de Chile, respectivamente). Dentro de sus intereses se encuentra la fotografía y observación de aves.

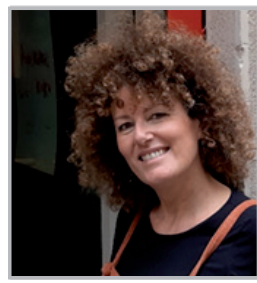

\section{Blanca García Henche}

Blanca.garcia@uah.es

Facultad de CCEE y Turismo. Universidad de Alcalá

Blanca García Henche es doctora en Ciencias Económicas y Empresariales y profesora e investigadora del Area de Comercialización e Investigación de Mercados de la Universidad de Alcalá, especialista en Marketing Turístico. Sus principales campos de investigación son el turismo experimental y el marketing de destinos culturales. Sus investigaciones han sido publicadas en varias revistas académicas (C. de Turismo, Conservar Patrimonio, Frontiers in Psychology o Sustainability ) y en libros y capítulos de libro de editoriales de prestigio académico (ESIC-Pirámide, AGE, Marcial Pons, CISS Wolters Kluwer, etc.). Participa activamente como expositora en diversos congresos de carácter internacional centrados en temas de Turismo, Patrimonio, Cultura y Urbanismo (INVTUR, INCUNA, AEDEM O CITURDES.) Ha prestado servicios de consultoría sobre liderazgo y marketing turístico a organizaciones públicas y privadas como Promotur, TurEspaña o Ayuntamiento de Madrid. 